\title{
Distraction Osteogenesis of Mandible in Hemifacial Microsomia-A Case Report
}

\author{
*Simon Chummar and Bhushan Jayade \\ Dental Department, NMC Specialty Hospital, UAE \\ Submission: May 17, 2016; Published: May 30, 2016 \\ *Corresponding author: Simon Chummar, Dental Department, NMC Specialty Hospital, PB-7832, Dubai, UAE Tel: +971502010266; Email: \\ drsimash@rediffmail.com
}

\begin{abstract}
Distraction osteogenesis is a process of generating new bone by slowly stretching the callus. Mandibular distraction osteogenesis has gained popularity as a technique for managing patients with mandibular hypoplasia. The reconstruction of severe mandibular deformities in patients with hemifacial microsomia (HFM) is difficult. We report a case of distraction using an intraoral approach for the corticotomy and an intraoral bone lengthening device. Our case was of Hemifacial Microsomia Pruzansky type 2a.
\end{abstract}

Keywords: Distraction osteogenesis; Hemifacial microsomia

\section{Introduction}

This concept of bone lengthening was first described by Codivilla in 1905, who used it to elongate a femur by repeated distraction forces [1]. Other surgeons also applied the technique, but met with failures due to complications such as nonunion, nerve damage, local edema, skin necrosis, and pin tract infection. But Ilizarov in Russia developed and popularized this technique of bone lengthening by gradual distraction which was applied to the enchondral bones of the upper and lower extremities. Lengthening of the mandible and midface has been described in several animal studies. McCarthy et al first described lengthening of the human mandible by distraction osteogenesis using an extra oral approach. We report a case of Hemifacial Microsomia Pruzansky type 2a. In which distraction was done by intraoral approach for the corticotomy with an intraoral AO mandibular distractor.

\section{Case Report}

Miss U.R aged 10 years reported to the department of Oral and Maxillofacial surgery at NMC Speciality Hospital with a previous diagnosis of Hemifacial Microsomia Pruzansky type 2a (Figure 1). She had undergone Costocondral grafting at the age of 4 years on the left Temporo mandibular region. She had deranged occlussion with severe cross bite, inability to chew food, midline shift to left, poor oral hygine. and lack of fullness of cheek on left (Figure 2). 3D CT scan showed hypoplasia of maxilla and mandible left side with rudimentary left condyle and coronoid (Figure 3). It was decided to elongate the mandible on the left so we did intraoral corticotomy at the mandibular angle and fixed an intraoral AO distractor (Figure 4). The points considered while placing the distractor are the occlusal plane, the roots of teeth, planned vector of distractor, location of the mandibular nerve, adequate bone for screw placement. Lip closure and location of activation hex. A $5 \mathrm{~cm}$ long incision was then made on the inferior buccal sulcus along the ascending ramus and the periosteum elevated. The procedure involved by adapting the foot plates of the distractor to the mandible and initially two screws are placed which are not fully tightened on either side of the intended corticotomy. The screws are removed and the distractor removed from the mouth. The corticotomy is done taking care not to complete the cut and protecting the mandibular nerve. The distractor is brought back in to the mouth and fixed with screws, with a minimum of three screws on each foot plate. The cut is completed with an ostetome and the distractor is activated to move the bony segments apart, it should be freely movable. The distractor is brought back to passive state and the wound is sutured. After a week of consolidation the distractor is activated at the rate of $1 \mathrm{~mm} /$ per day till the over correction of mid-line was achieved by $3 \mathrm{~mm}$. Once this was achieved the distraction was stopped and a consolidation period of four weeks was given before the distractor was removed. Distraction of $13 \mathrm{~mm}$ of bone was achieved (Figure 5). Simultaneously traction was given in the direction of distraction using arch bars and elastics. 

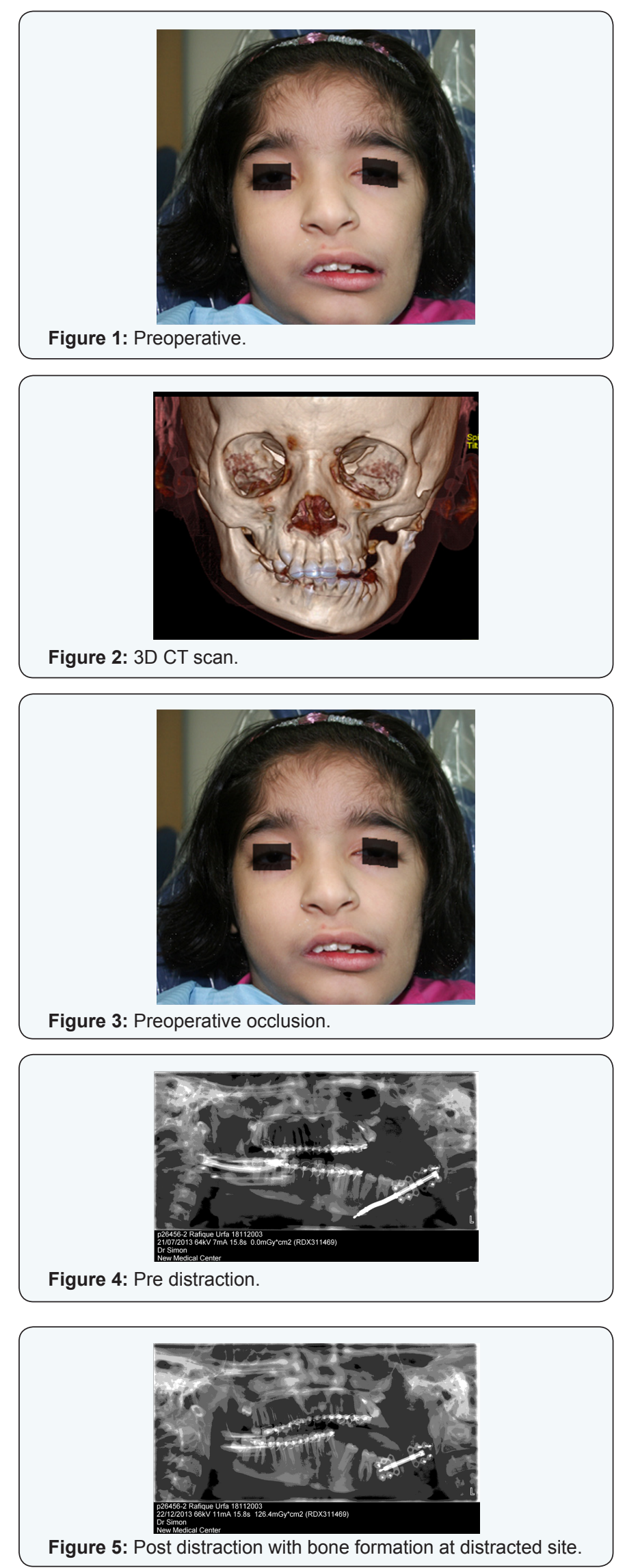

\section{Discussion}

McCarthy et al first described lengthening of the human mandible by distraction osteogenesis using an extra oral approach [2]. In his technique a corticotomy on the buccal and lingual aspect of the mandible was done, with preservation of the periosteum and the intramedullary blood supply. The distraction device is mounted on both sides of the corticotomy and a latency period of 5 to 10 days is allowed for primary callus organization. Then gradual distraction is performed at the rate of 0.5 to $1.5 \mathrm{~mm}$ per day. After the desired lengthening has been achieved, a period of consolidation of a few weeks is suggested before removal of the device. In our case we used intraoral approach for corticotomy and distractor placement with the extension arm of distractor extruding from the mental foramen region on skin. The distraction in our case was started after 1 week of consolidation and was done at the rate of $1 \mathrm{~mm} /$ per day. Post distraction after consolidation of callus for a period of four weeks we removed the distractor.

Distraction Osteogenesis can be performed at any age. When mandibular distraction is used early in childhood, the maxilla is released from the constricting effect of the short ascending ramus and the masticatory muscles [3]. The lateral open bite was corrected by vertical growth of the maxilla. Simultaneous maxillo-mandibular distraction is necessary after school age. Our patient was 10 years old and the lateral openbite is planned to be corrected by orthodontic treatment.

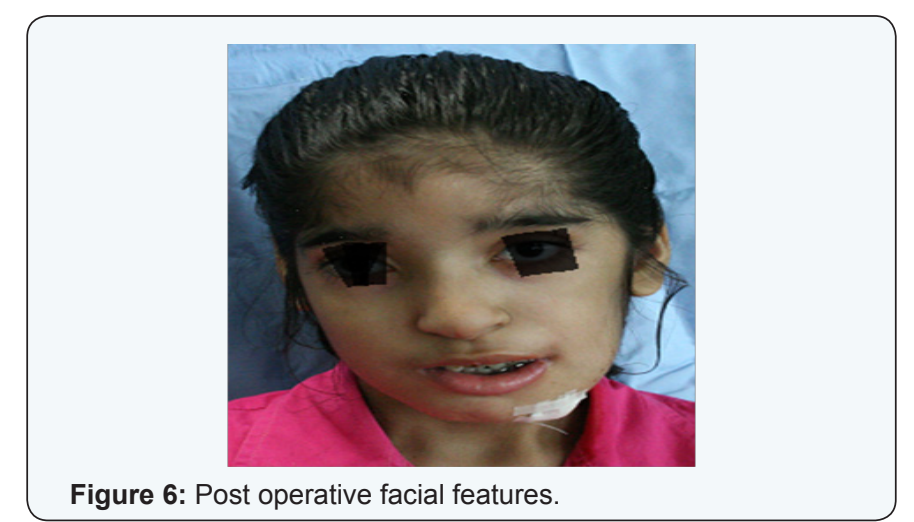

In selected patients, mandibular distraction osteogenesis can be beneficial by improving overall facial symmetry and balance (Figure 6). Mandibular distraction osteogenesis has unique advantages for these patients in that it can be performed early in childhood with minimal morbidity [1]. In this method the soft tissue attached to the bone is expanded simultaneously. The main advantage of this procedure is the elongation of the muscles, ligaments, vessels, nerve, subcutaneous fat, and skin that could not be achieved by other radical procedure [3]. According to Maria Costanza Meazzini el. al. although shortterm changes obtained with unilateral distraction osteogenesis of the deficientSascending ramus of the mandible are extremely satisfying in hemifacial microsomia patients, the genetic input 
on neuro-muscular and skeletal architecture tends to slowly mould the distracted skeleton back to the original configuration [4]. Honest communication with the families and professional psychological support are mandatory in planning the treatment of young patients affected by hemifacial microsomia. Our patient needs long term followup to know if the change achieved by distraction is stable. Our patient is undergoing orthodontic treatment and may require surgical intervention to correct the residual deformity after completion of growth.

Some authors recommend orthopedic forces which allow for a more stable occlusal plane and for a slower return of the mandibular vertical asymmetry, but it have mainly a dentoalveolar effect [5]. Therefore, the decision of applying an orthopedic force associated with distraction should be taken by surgeon and orthodontist together, taking in to consideration the advantages and the disadvantages of this treatment. In our case orthopedic force was applied using arch bars and elastics in the direction of distraction (Figure 7).

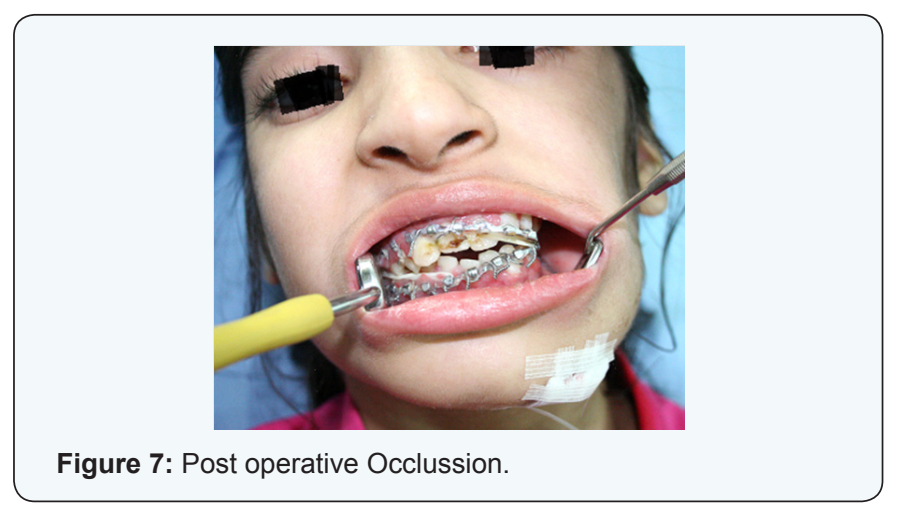

\section{Conclusion}

Distraction Osteogenesis holds promise for early reconstruction of craniofacial skeletal defects without the need for bone grafts, blood transfusion, or intermaxillary fixation, but long term follow up is needed to see if results achieved by distraction is stable [6]. No bone grafting is required but more patient coperation is required. Distraction allows normal function during the procedure, hence functional remodelling of the callus and surrounding tissue takes place. In patients with syndromic craniosynostoses, distraction can be applied at strategic times as part of a staged surgical treatment plan for the management of severe skeletal discrepancies. Distraction may be regarded as a useful additional technique to minimize skeletal deformities.

\section{Acknowledgment}

Special thanks to Prof. Dr. Ulrich Joos, Dr. Sudhakar Tellicherry (Anesthesia), Dr. Vickrant Malhotra (Radiologist) Dr. Sachin Mudrale (Orthodontist), Dr. Ramesh Pillutla (Radiologist).

\section{Reference}

1. Rachmiel A, Levy M, Laufer D (1995) Lengthening of the mandible by Distraction Osteogenesis: Report of cases. J Oral Maxillofac Surg 53(7): 838-846.

2. Polley JW, Figueroa AA (1997) Distraction osteogenesis: its application in severe mandibular deformities in hemifacial microsomia. J Craniofac Surg 8(5): 422-430.

3. Monasterio FO, Molina F, Andrade L, Rodriguez CDDS, Arregui JS (1997) Simultaneous Mandibular and Maxillary Distraction in Hemifacial Microsomia in Adults: Avoiding Occlusal Disasters. Plastic \& Reconstructive Surgery 100(4).

4. Meazzini MC, Mazzoleni F, Bozzetti A, Brusati R (2012) Comparison of mandibular vertical growth in hemifacial microsomia patients treated with early distraction or not treated: Follow up till the completion of growth. J Craniomaxillofac Surg 40(2): 105-111.

5. Meazzini MC, Mazzoleni F, Bozzetti A, Brusati R (2008) Does functional appliance treatment truly improve stability of mandibular vertical distraction osteogenesis in hemifacial microsomia? J Craniomaxillofac Surg 36(7): 384-389.

6. Meazzini MC, Mazzoleni F, Bozzetti A, Brusati R (2005) Mandibular distraction osteogenesis in hemifacial microsomia: Long-term followup. J Craniomaxillofac Surg 33(6): 370-376. 